\title{
From Chalk Talk to Tablet Talk: Pedagogies for Control Engineering *
}

\author{
D.I. Wilson* P. Maclaren ** \\ * Electrical \& Electronic Engineering, \\ Auckland University of Technology, Auckland, New Zealand \\ (e-mail: david.i.wilson@aut.ac.nz) \\ ** Centre for Learning and Teaching, Auckland University of \\ Technology, Auckland, New Zealand, \\ (e-mail: peter.maclaren@aut.ac.nz).
}

\begin{abstract}
Individual disciplines, and even subfields within those disciplines, typically exhibit distinctive pedagogical approaches that have developed over time, influenced by the content of the discipline and technology. In control engineering, mathematical symbolic and diagrammatic forms are a key element, and have been traditionally taught using oral and handwritten approaches (so called chalk-talk). The widespread use of computer display technology and PowerPoint has influenced the way in which the development of ideas is presented, leading to mathematics presented as solutions, rather than a process, and the isolation of the mathematics from the control engineering context. This paper reviews educational trends and personal experience that suggests the use of digital pen-enabled tablet technologies can facilitate the reintroduction of elements critical to developing an effective pedagogy for control engineering.
\end{abstract}

Keywords: control education, Tablets, chalk talk, signature pedagogy, design-orientated, flipped classroom

\section{INTRODUCTION}

Within disciplines, distinctive styles of teaching have evolved that reflect the particular nature of that discipline's content and its associated technologies. While some generic methods of teaching may be observed in use across a range of disciplines, these methods are often manifest in particular ways within different disciplines.

Lee Shulman coined the term signature pedagogy to denote the distinctive approaches that occur within individual professions suggesting that signature pedagogies don't just reflect the nature of the discipline, but they help shape it; that signature style was pervasive, (cutting across courses and institutions), entrenched (current academics were taught in a similar style a generation previously), and largely successful, Shulman (2005).

It is interesting to note that Shulman uses 'robust' and 'durable' to describe these signature pedagogical styles, using terminology that control engineers would use to describe control loops that ensure the stability of a system.

In this paper we will present our view of how teaching of engineering, in the specific instance of advanced control engineering, has evolved, and how it might develop in the future through the use of new technologies. The paper does not concern itself with what topics should be taught in a controls course, what constitutes a suitable support laboratory program, nor even argue the merits of the var-

\footnotetext{
* Financial support from the School and Engineering and from the Centre for Learning and Teaching, Auckland University of Technology, New Zealand is gratefully acknowledged.
}

ious CACE packages - issues that were comprehensively surveyed in Kheir et al. (1996). This paper is concerned with the how of teaching in control courses, with Optimal Control, a joint final year elective and Master's paper providing the context.

\section{CHARACTERISTICS OF CONTROL ENGINEERING PEDAGOGY}

\subsection{A control engineering approach to learning and teaching}

If we look at different pedagogical approaches to the teaching of control, we can identify components that reflect the discipline of control. Feedback loops are a critical component in both educational theory and control engineering.

Abdulwahed and co-workers at Loughborough, Abdulwahed et al. (2008), redrew Kolb's learning cycle as a feedback loop familiar to all control engineers. Their focus is on knowledge acquisition: the reference input is the knowledge to be learned, the output is the actual learned knowledge, and the observation (presumably through examinations) adjusted the knowledge transformation experience. In fact the analogy is pushed even further with the assertion that the input may be corrupted with noise and the plant (modelled as an integrator) may exhibit uncertainties, and the role of the controller. From this it is but a small step to hypothesise the notion of stability, robustness under uncertainty etc, all concepts that are well established in control theory. 
Laurillard's Conversational frame work provides a more complex model of the learning and teaching process, Laurillard (2002). It identifies a critical need for students to be able to test their understanding of concepts against the understanding of the lecturer, and to access feedback that can develop their understanding appropriately. The lecturer also needs to be able to modify their teaching approaches in response to feedback on apparent deficiencies in student understanding. The teaching approaches used in control education needs to provide mechanisms through which teacher and student can develop and explore their common understandings within mathematical and engineering contexts.

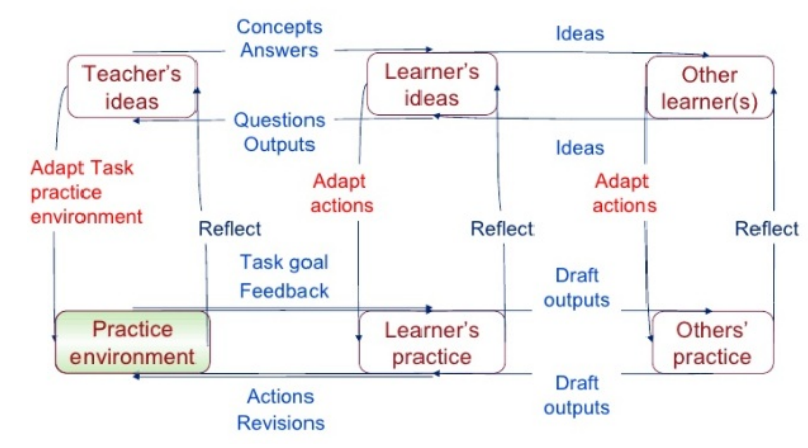

Fig. 1. What it takes to learn: the 'conversational framework' for the teaching-learning process. Figure adapted from Laurillard (2002).

\subsection{Mathematics in Control Engineering}

With mathematics being a core component of engineering, it is unsurprising that approaches that are distinctive within mathematics are also present in control engineering. The method of problem solving exposition described as 'chalk talk' by Artemeva and Fox (2011) has played a central role in many mathematics based disciplines.

It is important here to distinguish Artemeva and Fox's chalk talk, specific to mathematical disciplines, from the generic 'chalk and talk' method commonly referred to in educational contexts. This generic chalk and talk approach is often used disparagingly to describe a lecturer-centred, didactic mass lecture approach that lacks interactivity and student engagement, Mills and Treagust (2003), Armbruster et al. (2009).

On the other hand, chalk talk is a particular mathematics specific genre of teaching that involves interactive modelling of the solution development process using handwritten mathematics accompanied by a verbal commentary, usually within a structure providing for progressive, hierarchical knowledge development. Artemeva and Fox describe chalk talk as a "situated disciplinary practice" and contend that it "can also be pedagogically interactive, meaningful, and engaging as a way into disciplinary doing and being".

Even while advocating alternatives to lecture based approaches, such as problem or project based learning, Mills and Treagust note that "mathematics, physics and much of engineering have a hierarchical knowledge structure" such that more traditional approaches to the development of fundamental skills have value, particularly in initial stages of knowledge development. Chalk talk can play a vital role in control engineering education.

While Artemeva and Fox focus on the chalk talk approach in the context of the mass lecture, this process is also apparent in contexts with fewer participants, such as tutoring sessions involving a single student and using pen and paper, or even between students sharing ideas in a problem solving context. We would suggest is that chalk talk has a role to play in control engineering whatever the wider approach.

In a mass lecture theatre there are clearly skills and strategies that need to be used to engage a larger audience. Artemeva and Fox refer to 'the cinematic art of teaching university mathematics' and note that a certain amount of theatrics that may be required to maintain student involvement.

This paper looks at the use of chalk talk as it has been manifested in our teaching environments. It examines how changes in classroom technology have restricted the use of the chalk talk genre, and how the introduction of new technology, in the form of the Tablet PC, may revitalise the genre. We will also suggest that technology developments can enable new applications of this genre in alternative teaching and learning contexts.

\subsection{The value of sketching in Control Engineering}

While control engineering, as a mathematically intensive discipline, includes chalk talk as a component, we would argue that giving an emphasis to the engineering context to the problem solving process adds another essential element. This requires a distinctive adaptation to the standard chalk talk approach; as described by Kheir et al. (1996), there are two streams of control: the mathematics of the process, and the actual thing to be controlled.

Spending too much time on the mathematical minutiae of the process (the exacting proofs, the pathological cases, the effect of right-hand plane zeros etc) while ignoring the real application of the process is disconcerting to the average student; it can lead to a view of lecturers as academic pedants rather than practicing engineers. After all we should not forget that the subject of control engineering has had a controversial recent history, notably the Lund/MIT differences in the robustness of then then popular field of adaptive control, and the somewhat belated acceptance from Academia of the genuine usefulness of model predictive control. In both cases the controllers clearly worked in real situations and enjoyed strong industrial support, but some still cautioned that rigorous mathematical proofs of stability had not been produced, Anderson (2005).

One way to facilitate bridging the two streams of control is to resurrect the importance of drawing and sketching in the design process, and integrate it with the mathematics. The context for the process can then revealed concurrently with the mathematics.

Mechanical engineering educators have long recognised the value of sketching with Ullman noting that "engineers are notorious for not being able to think without making 'back-of-the-envelope' sketches of rough ideas", Ullman 
et al. (1990). While Ullman's work focussed essentially on product design, the study drew parallels between problem solving and drawing, highlighted the richness and communication possibilities in sketching, and finally noted the key differences between sketching and formal drafting. Writing in 1990, they were conscious of the then limitations of trying to sketch figures in a computer aided environment. The ' $\mathrm{D}$ ' in CAD, they asserted, stood for drafting, not design. The medium subtly changes the message.

In the same way that presenting mathematics in a dynamic handwritten form assists in understanding mathematical developments, we would suggest that sketching can assist in understanding the engineering context. An environment that can integrate handwriting and sketching can facilitate the development of a discipline specific variation of chalk talk.

In teaching control engineering, it is apparent that previous developments in educational technology have tended to work against the use of both handwriting and sketching, and have fostered the use of more formal preformed diagrams and text.

\subsection{The History of the Teaching Experience}

Prior to 2012, the paper Advanced Engineering Maths a pre-requisite for our advanced control papers, with almost 100 students was delivered using a fairly classical largelecture style model. Optimal Control, an elective with only 5 undergraduates and 8 master's students, was run more in a relaxed conversational manner.

However, in both cases, pre-prepared PowerPoint slides had become a primary delivery mechanism. With timetable allocation primarily carried out at an institutional (rather than school or discipline level), teaching spaces are no longer tailored to the needs of specific disciplines. The fact that the datashow has been adopted as an institutional standard across classrooms and lecture theatres, and that suitable whiteboard space is no longer a given, there has been an influence for moving from traditional chalk talk pedagogy to the use of PowerPoint presentations.

Pre-prepared PowerPoint slides, detailing all the mathematical derivations, examples and even code snippets, had become the standard. Over the years, the overheads and support material were gradually updated and modified; additional material was added successively to provide mathematically consistent, self-contained and correct versions of various special cases.

In reality the situation became increasingly unwieldy; the overheads started to resemble a textbook, and the lecture lost its focus whilst simultaneously the university administrators shortened the teaching semester. In order to cover this new material (all of it considered vital at the time), the lecturer needed to rapidly run through the slides. The pre-set structure meant that there was less sensitivity to student reaction to the material, and less capability to respond by modifying pace and reinforcing content as appropriate to student needs.

There was increasing absence of students from lectures; without the feedback in the lecture, students saw limited value over what could be acquired by referring later to copies of the slide notes. There was an increased dropout rate; there was arguably less engagement with the material, and less opportunity for students to ask questions to clarify points at the time when they were first encountering problems. The essential value of the lecture in facilitating questions and answers from students and lecturer was lost; the feedback loop was broken.

\subsection{The Role of the Text}

As the slides developed in complexity and detail, they began to resemble the textbook, so the students might just as easily refer to the text. What then of the sufficiency of the textbook as a primary educational resource? The presentation of the mathematics of control engineering in text book usually takes the form of carefully formatted and structured solutions; the more fuzzy nature of the problem formulation, the relationship to engineering context, and the dynamic process of solution are less easily represented.

A quick perusal through many advanced control texts (such as those surveyed in Kheir et al. (1996)) will highlight a revealing omission: there are very few engineering diagrams, especially sketches. This is particularly true for the advanced texts where the literally thousands of elegantly presented equations are sporadically broken up with formal block diagrams and the resultant Matlab plot.

\subsection{Current Approaches - in Summary}

While Schulman has argued for the robustness of signature pedagogies, he also noted that changes in technology could have an impact, Shulman (2005). In fact, the changes from whiteboards to data projectors as the standard classroom technology have over time led to significant changes in pedagogical approaches in control engineering. We had moved from the use of interactive chalk talk approaches, to using static pre-prepared PowerPoint slides in the lecture context. This has led to the modelling of the thinking process being largely absent. Textbooks also emphasise the polished solution, at the expense of an understanding of process.

\section{3. (RE) DEVELOPING A LEARNING AND TEACHING APPROACH WITH NEW TECHNOLOGY}

Given these developments, in 2012 a new approach was sought to redefine the role of the lecturer and the classroom experience. We wanted to (a) reinvigorate the class experience, (b) decrease the mathematical minutiae and increase the art and flair of the subject, and (c) improve the learning by stressing the holistic importance of control.

Tablet PC devices were seen as offering the possibility of a return to the use of dynamic chalk talk approaches, while building on the strengths and universal availability of the digital environment of data projectors (and accommodating the diminishing availability of whiteboard space). Using a digital pen enabled tablet, the lecturer can develop the graphic approach on a smaller scale, while projecting at a large scale; the digital environment allows the lecturer to seamlessly scroll backward and forward and to zoom to emphasise points. 
To maintain the conversational style while developing mathematical reasoning, the digital device needs to be able to support a natural writing and drawing experience. The inbuilt digitiser of the Tablet PC supports a high degree of resolution and precision that allows detailed drawing and fine writing, and pressure sensitivity that emulates pen on paper. The MS Windows PC operating system supports the stylus capability across the range of MS Office and third party software. The Tablet PC has been widely and successfully used as a teaching tool, and currently continues to be the most appropriate for the development of a digital chalk talk approach, Amelink et al. (2012).

In 2012, five HP Tablet PCs were made available to a group of engineering lecturers for classroom use through an AUT University LATENT (Learning and Teaching ENabled by Technology) Grant managed by the Centre for Learning and Teaching.

While a range of collaborative software options were possibilities, MS OneNote was adopted as the primary 'whiteboard' delivery platform for the Control Engineering courses. While PowerPoint has some inbuilt support for annotation using pen tools, it was not as flexible as OneNote. We also wanted to move away from the previous environment, and discourage the tendency to hurriedly flash 100 pre-prepared slides in the 50 minute lecture.

\subsection{Teacher Experiences}

Atemeva and Fox reference the multimodal nature of chalk talk, with experienced lecturers unconsciously integrating whole-body and hand movements with their commentary. Just as presenting on a whiteboard (or chalkboard) requires development of these particular skills, presenting via a pen-enabled monitor requires development of a new set of strategies and actions.

The scale of the device changes the dynamics of the presentation. The lecturer must still maintain an element of the theatrical to sustain the dialogue and the interest; competence with the technology must be developed; slick transitions are vital important.

The first most visible change is that the teacher is now continuously facing the students. Even better, with a wireless connection to the data monitor, the teacher is able to wander around the class, perhaps soliciting ideas from the students. This immediately addresses one of the key less desirable characteristics in that Shulman noted; that of the engineering students continually facing the back of their lecturer.

Clearly, writing on the small screen of the Tablet PC is not the same as writing on a white board. In a related project, Maclaren et al. (2012), some teachers found that considerable adaption was required in planning a lesson, in what to write, where to write and how to write, to maintain the level of presentation that had they had evolved in many years of teaching on a whiteboard.

The change of scale in the writing space does not just require physical adaption in writing dynamics but can result in changes in the dynamics of classroom interaction. In a chalk talk approach with whiteboard, many gestures related to the activity directly on the board as it was added. When using the Tablet, students are focussed on the large screen, rather than on the lecturer who is primarily involved with the tablet monitor. While the lecturer might emphasise material by highlighting on the tablet, in other instances the lecturer might use gestures in front of, and with direct reference to, the projected screen. The effectiveness of different approaches in this environment is something that lecturers will continue to develop and evaluate.

In teaching Optimal Control, the lecturer made a deliberate attempt to not replicate the course text, but rather explore the possibilities that a text book could not offer; namely the use of colour, sketching, and the importance of the accompanying voice, what Artemeva and Fox (2011) refer to as metacommentary.

Fig. 2 shows a screenshot from the Optimal Control course. The material discussed here is well suited to a graphical presentation, and, what is not so clear in the figure, is that the classroom clarity is significant improvement over a whiteboard. For example, one of the authors struggled to find a suitable diagram supporting the concept of Lagrange multipliers in optimal control. The 2D single colour diagrams common in many texts (perhaps due to publishing economic constraints) can be hard to interpret, but can be sketched on a tablet. Again the careful use of colour combined with the fact that the lecturer is drawing the sketch in realtime while supplying a metacommentary provides what is essentially an animation. Note however that this animation requires no special skills nor expense to produce.

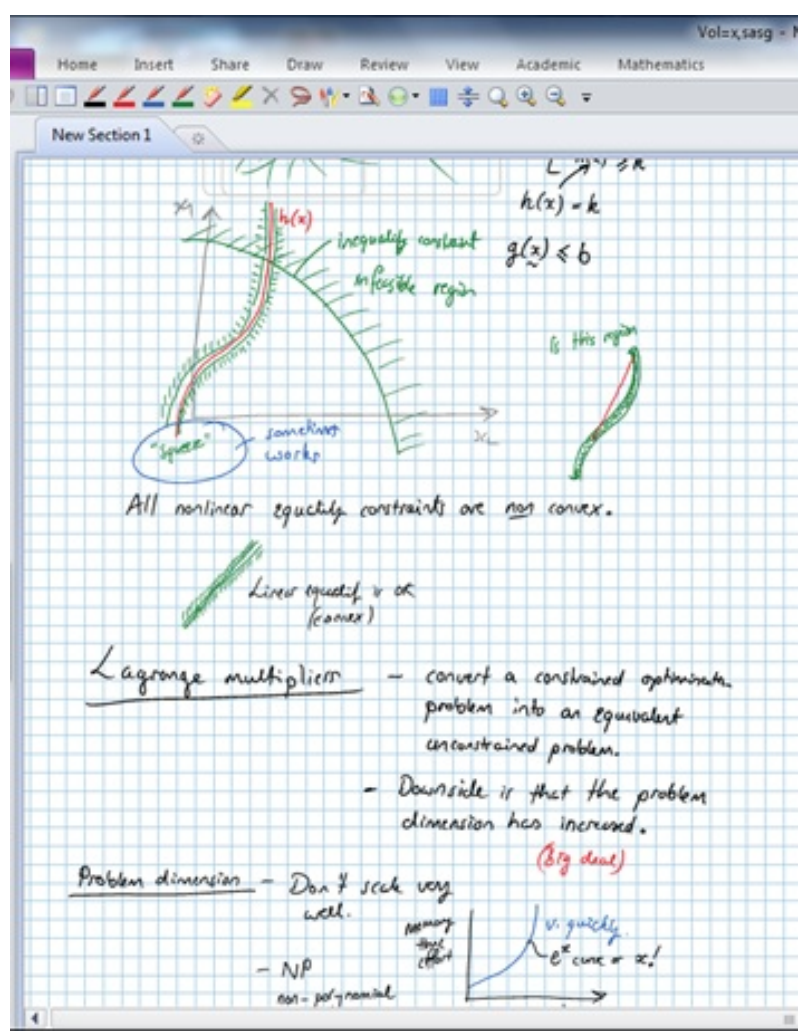

Fig. 2. Optimisation notes as delivered in class using OneNote.

The strait-jacketing enforced by Powerpoint to fit a coherent subsection on a single slide is removed because 
one can write on a continuous stream of paper (a 'toilet roll' approach). Using OneNote, the lecturer can zoom and scroll around, and if done carefully, the students can follow the details, but still retain a holistic view of the problem. Of course given the density of information within mathematics, a data projector cannot compete with a large blackboard. Artemeva and Fox (2011) quote mathematics professors speculating on the ideal blackboard (large and surrounding all four walls!) and stressing the planning and choreography required to develop a complex mathematical argument.

While the handwriting in Fig. 2 shows the author is not a draftsman, the fact that it is informal, complete with mistakes and the subsequent corrections, means that it more closely models the process that students themselves will need to undertake. Students appeared to appreciate and intuitively recognise this as important. Although the small size of the writing space on the Tablet requires adaptation from the lecturer, it does mean that the lecturer is writing on a canvas that is of similar dimensions to that of the student, which may help the student adopt appropriate layouts.

\subsection{Using colour to aid understanding}

A systematic and deliberate use of colour is now possible given that the Tablets offered a rich set of vivid colours that are difficult to replicate even on a white board. For example, we have found it useful when discussing recursive least-squares estimation in our Adaptive Control courses to colour the (cold) inputs blue, the (hot) outputs red, and the (natural) noise terms green. Consequently a discrete model is coloured as

$$
A(q) y_{k}=B(q) u_{k}+C(q) e_{k}
$$

This colour convention is continued in the diagrams. For example the upper figure in Fig. 3, not only colour-codes the output and input variables, but also uses vivid colours for the recent historical values with a gradual change to muted pastel shades for the older, less important, data. An added advantage is that such a colour scheme subconsciously prepares the students for concepts like the forgetting factor in RLS schemes where the older data is both faint and gradually forgotten.

The lower figure graphically illustrates the matrix inversion lemma used to convert an offline identification to an online recursive strategy. Again the pink blocks represent old data assembled in blocks of which we know the inverse, whereas the dark red blocks are the new updated component which will modify slightly our previous estimate. By using coloured blocks, both the mathematical development, and more importantly, the rational behind it, is easier to follow. In situations where we do not have a standard colour convention, we use black for the 'standard maths', but superimpose often at an oblique angle, a blue colour for the commentary (describing about the development), and green describing small notes and cautions.

We have tried to use this colour convention across all our control courses. Consequently the lecture overheads, even the course textbook, Wilson (2013), must now be reproduced in full colour. Given the prevalence of studentowned Tablets or laptops in the classroom, this colour

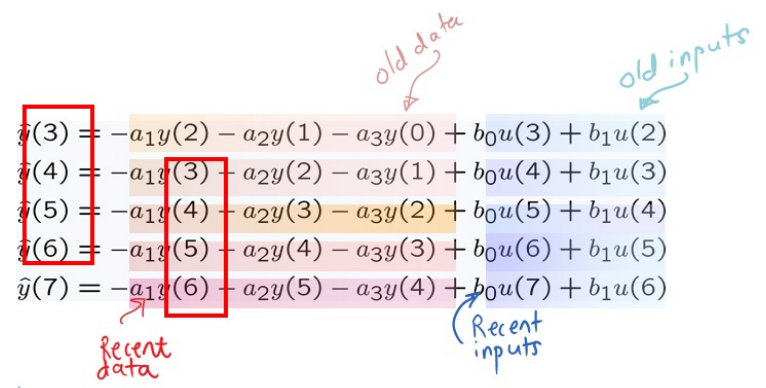

(a) The use of colour and annotations on pre-prepared PowerPoint slides. In this case the outputs are red and inputs are blue with the older data in less vivid pastel shades.

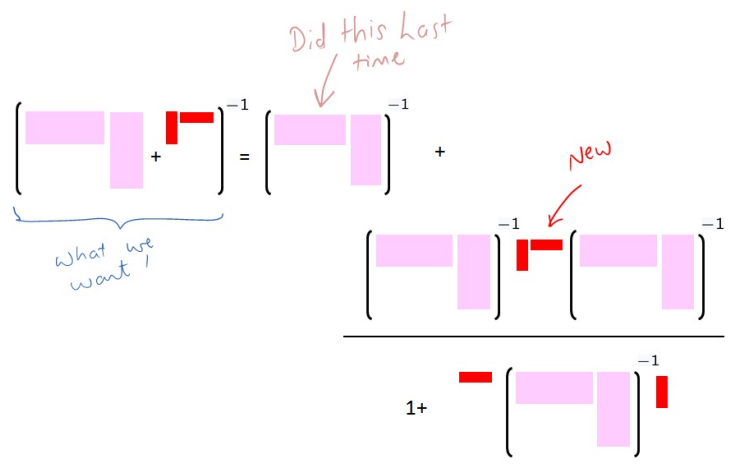

(b) Explaining the matrix inversion lemma using only differing coloured blocks. The vivid red blocks stem from the recently acquired input/output data.

Fig. 3. Using colour, shading and sketches to aid understanding for control concepts.

constraint is less of an economic burden than even just a few years previously. Of course one can use colour on whiteboards, or at a stretch, even blackboards. Practically though, the colours are limited, and the interruption when changing felt pens or chalk disrupts the dialogue and diminishes the theatre. Hence it tends not to happen. With tablet s though, it is easy to adjust colour, thickness and pressure in a natural way. Finally, it is interesting to hear the click of pens throughout the classroom as the majority of the students willingly follow my conventions.

Working in the digital environment means that other programs are directly accessible for immediate viewing via the same projector. The issues of switching between whiteboard and projector, with accompanying lighting changes are avoided. Demonstrations of software are immediately accessible, and output can be readily included in the lesson, and then annotated or highlighted in colour.

\subsection{Student feedback}

The response of students to the use of the Tablet PCs was overwhelmingly positive. In an informal pilot survey of 77 students, almost all agreed that the approach was better than other presentation approaches (i.e PowerPoint), and were keen to see the use extended to other papers.

Students commented on both functional and motivational improvements. Functional improvements included: the improved visual clarity of the material, and unobstructed 
visibility from anywhere in the room (vs whiteboards); improved aural clarity, with the lecturer facing the students, not the board. Students also noted their increased engagement with the material, and the lecturer (vs pre-prepared PowerPoint); students commented that they could see all the steps required/executed, and could review them, and not just see a completed example/answer. They saw a benefit in slowing down the lecture and covering the material one step at a time rather than displaying a PowerPoint slide with 'lots of words which can be hard to follow' They commented on the effectiveness of the approach in facilitating notetaking in conjunction with the lecturer, describing it as encouraging 'an active learning approach'.

The comments of the students support this use of technology as both technically better and more engaging. This informal feedback echoes the reports from other recent studies of the potential benefits of the use of Tablet PC technology such as Ambikairajah et al. (2007) or Olivier (2005).

It is of note that many of the advantages that students identified can be directly related to the return to a chalk talk genre of delivery. This suggests that taking chalk talk into a digital environment can enhance the learning experience while still maintaining the benefits of the genre.

\section{FUTURE DEVELOPMENTS}

While this project has been introduced in the context of a teaching model based around traditional lectures, the use of the Tablet is also seen as appropriate for providing the benefits of chalk talk using other models. For example, the 'flipped classroom' approach (see for example Zappe et al. (2009)), is one we are seeking to develop in this context. Tablets can be easily used to record screencasts to provide out-of-class access to chalk talk style examples of problems solving methods, Berger (2007). Further opportunities for using Tablet PCs in collaborative workspaces are opened up when students as well as lecturers have access to Tablet PCs, Hamilton and Hurford (2007).

\section{CONCLUSIONS}

The chalk talk genre provides an essential and authentic introduction to the processes of problem solving in mathematics and engineering. Many teaching environments no longer provide suitable whiteboard spaces for this approach and the lecturer can become constrained by the requirement to use digital projection and Powerpoint. The use of pen-enabled Tablet PCs allows a return to a dynamic handwritten approach and allows drawing and sketching in vivid colour. The initial feedback from students suggests that this is an approach that has the potential to engage students and produce better learning outcomes.

\section{ACKNOWLEDGMENTS:}

The authors wish to acknowledge the contributions of the project team members, especially Dr Sarat Singamemni and the contributions of the AUT project team.

\section{REFERENCES}

Abdulwahed, M., Nagy, Z.K., and Blanchard, R. (2008). Beyond The Engineering Pedagogy: Engineering The Pedagogy, Modelling Kolb's Learning Cycle. In 19th Annual Conference of Australasian Association for Engineer Education. Yeppoon, Australia.

Ambikairajah, E., Epps, J., Sheng, M., and Celler, B. (2007). Tablet PC and Electronic Whiteboard Use in Signal Processing Education. IEEE Signal Processing Magazine, 24(1), 130-133.

Amelink, C., Scales, G., and Tront, J. (2012). Student use of the Tablet PC: Impact on student learning behaviors. Advances in Engineering Education - A Journal of Engineering Education Applications, 3(1), 1-17.

Anderson, B. (2005). Failures of adaptive control theory andtheir resolution. Communications in Information and Systems, 5(1), 1-20.

Armbruster, P., Patel, M., Johnson, E., and Weiss, M. (2009). Active learning and student-centered pedagogy improve student attitudes and performance in introductory biology. CBE Life Sci Educ, 8(3), 203-213.

Artemeva, N. and Fox, J. (2011). The writings on the board: The global and the local in teaching undergraduate mathematics through chalk talk. Written Communication, 28(4), 345-379.

Berger, E. (2007). Podcasting in engineering education: A preliminary study of content, student attitudes, and impact. Innovate: Journal of Online Education, 4(1).

Hamilton, E. and Hurford, A. (2007). Combining collaborative workspaces with tablet computing: Research in learner engagement and conditions of flow. In 37th Annual Frontiers In Education Conference, T3C-3-8.

Kheir, N., Astrom, K., Auslander, D., Cheok, K., Franklin, G., Masten, M., and Rabins, M. (1996). Control systems engineering education. Automatica, 32(2), 147-166.

Laurillard, D. (2002). Rethinking University Teaching. A conversational framework for the effective use of learning technologies. Routledge, London.

Maclaren, P., Singamneni, S., and Wilson, D.I. (2012). Technologies for Engineering Education. In 11th Global Congress of Manufacturing and Management, GCMM2012. Auckland, New Zealand.

Mills, J. and Treagust, D.F. (2003). Engineering education-Is problem-based or project-based learning the answer? Australasian Journal of Engineering Education, 3, 2-16.

Olivier, W. (2005). Teaching mathematics: Tablet PC technology adds a new dimension. In 8th International Conference on The Mathematics Education into the 21st Century Project, 176-181.

Shulman, L.S. (2005). Signature pedagogies in the professions. Dadalus, 134(3), 52-59.

Ullman, D.G., Wood, S., and Craig, D. (1990). The importance of drawing in the mechanical design process. Computer \& Graphics, 14(2), 263-274.

Wilson, D.I. (2013). Advanced Control Using Matlab or Stabilising the Unstabilisable. Auckland University of Technology, 3rd edition. 538pp.

Zappe, S., Leicht, R., Messner, J., Litzinger, T., and Lee, H.W. (2009). "Flipping" the classroom to explore active learning in a large undergraduate course. In Proceedings of the 2009 American Society for Engineering Education Conference and Exposition. Austin, TX, USA. 